\title{
SABERES Y ESCRITURAS FEMINISTAS EN CLAVE GENEALÓGICA: APROXIMACIONES A LA BRECHA DE MERCEDES VALDIVIESO
}

\author{
Carolina Escobar Lastra \\ Universidad Autónoma de Chile \\ Grupo de Investigación Literatura y Escuela \\ Talca, Chile \\ carolina.escobar@uautonoma.cl
}

\section{RESUMEN / ABSTRACT}

\begin{abstract}
Este artículo analiza La brecha (1961) de Mercedes Valdivieso, considerada la primera novela feminista de Chile. A partir de este antecedente, se busca problematizar los significados del feminismo que encarna el texto. Para alcanzar dicho propósito, se realiza, en primer lugar, una lectura en clave genealógica en torno a los principales acontecimientos e investigaciones sobre el rol de las prácticas literarias en la construcción de ideas feministas en Chile a partir del siglo XX. Posteriormente, a través de la puesta en diálogo con Los vigilantes (1994) de Diamela Eltit y Contra los hijos (2018) de Lina Meruane, se analizan los conceptos de cuerpo y maternidad, ejes articuladores del pensamiento de Valdivieso. Finalmente, se realizan algunas aproximaciones críticas sobre las aperturas y tensiones de La brecha en contraste con las emergencias de la política feminista reciente.
\end{abstract}

Palabras clave: genealogías, crítica literaria feminista, prácticas literarias feministas, feminismos del siglo XX, política feminista en el Chile reciente.

FEMINIST KNOWLEDGE AND WRITINGS IN A GENEALOGICAL KEY: APPROACHES TO LA BRECHA BY MERCEDES VALDIVIESO

This article analyzes La brecha (1961) by Mercedes Valdivieso, considered the first feminist novel in Chile. From this background, it seeks to problematize the meanings of feminism embodied in the text. To achieve this purpose, first, a genealogical reading of the main events and research on the role of literary practices in the construction of feminist ideas in Chile since the twentieth century is carried out. Subsequently, through the dialogue with Los vigilantes (1994) by Diamela Eltit and Contra los hijos (2018) by Lina Meruane, the concepts 
of body and motherhood, pivotal themes of Valdivieso's thought, are analyzed. Finally, some critical approaches are made on the openings and tensions of La Brecha in contrast with the emergences of recent feminist politics.

KEYWORDS: genealogies, feminist literary criticism, feminist literary practices, 20th century feminisms, feminist politics in recent Chile.

\section{INTRODUCCIÓN}

La brecha (1961) de Mercedes Valdivieso forma parte de un corpus de obras que, de acuerdo con su posicionamiento sobre las problemáticas sociales de las mujeres, pueden enmarcarse en un feminismo intencionado ${ }^{1}$. Dicha intencionalidad se evidencia en un discurso que recoge al menos tres grandes preocupaciones: el cuerpo, la maternidad y la crítica a los esencialismos del deber ser femenino. Estos abordajes han estado presentes en estudios precursores como los de Lucía Guerra, quien concibió la novela como irruptora y explícitamente feminista, destacando su lenguaje despojado de sublimaciones y eufemismos poéticos (Guerra, "Feminismo" 6), en contraste con otras autoras de los años cincuenta.

En esta misma línea, Olea se ha concentrado en la política escritural de Valdivieso, destacando sus aportes en una época de repliegue de los movimientos de mujeres tras la obtención del derecho a voto ("La palabra maldita" 102-103). Para la investigadora, La brecha abrió el signo de lo femenino a la pluralidad y heterogeneidad de lo público y activó un discurso de "ampliación de las identidades femeninas vigiladas desde los órdenes socialmente instituidos" ("La palabra maldita" 102). Esta ampliación, según sus interpretaciones, posibilitó la creación de "otros 'efectos de feminidad' [...] por el emprendimiento de una acción de políticas corporales, como el divorcio, el aborto, una segunda pareja y el ingreso al mundo del trabajo" (Olea, "Escritoras" 113); políticas y ejes temáticos que dan sentido al relato.

\footnotetext{
Esta idea forma parte de las reflexiones presentadas en la ponencia "Trayectos y tensiones de las representaciones del sujeto político del feminismo en las prácticas literarias de autoras chilenas recientes", XIV JALLA México 2020. "Feminismo intencionado" refiere a la serie de prácticas literarias que, de manera explícita, sitúan autoría y texto en dicho discurso.
} 
Lecturas recientes, como la realizada por Ana Inés Leunda, han analizado las estrategias de construcción de la heroína y las implicancias de las metáforas corporales presentes en el texto (69). Desde una perspectiva semiótica, concluye que en la construcción del mundo novelesco estas metáforas visibilizan "normas que hoy podemos calificar de sexistas y que tempranamente la conciencia creadora está denunciando" (Leunda 71). Esta conciencia ratifica el interés de Valdivieso por problematizar las estructuras que regulan lo femenino en los ámbitos socioculturales. Asimismo, destaca que el carácter feminista de La brecha se evidencia no solo en la rebelión de la protagonista ante los códigos patriarcales, sino también en la ausencia de un castigo frente a dicha rebelión. En este sentido, el relato recrea un mundo donde es posible existir en igualdad de condiciones, cuestión que Leunda interpreta como una apertura de caminos para las mujeres de su época (69). Esta táctica, también analizada por Guerra, permite, además, reconocer las alteraciones que la autora realizó a la norma textual de la época y a la verosimilitud aceptada que permitía una subversión inofensiva ("Feminismo" 7).

A partir de esta revisión crítica, en el presente artículo se busca problematizar los significados del discurso feminista que encarna el texto. Para alcanzar dicho propósito, se realiza, en primer lugar, una lectura en clave genealógica en torno a los principales acontecimientos e investigaciones sobre el rol de las prácticas literarias en la construcción de ideas feministas en Chile a partir del siglo XX. Posteriormente, a través de una lectura dialógica con Los vigilantes (1994) de Diamela Eltit y Contra los hijos (2018) de Lina Meruane, se analizan los conceptos de cuerpo y maternidad, ejes articuladores del pensamiento feminista de Valdivieso. Finalmente, se realizan algunas aproximaciones críticas sobre las aperturas y limitaciones de La Brecha en contraste con las urgencias y tensiones de la política feminista reciente.

\section{LAS PRÁCTICAS LITERARIAS EN LA PRODUCCIÓN DE CONOCIMIENTOS FEMINISTAS: UNA APROXIMACIÓN GENEALÓGICA}

En términos generales, las relaciones entre literatura y feminismo pueden ser abordadas desde distintos ángulos, de los cuales tres se consideran relevantes: la relación con las diversas instituciones literarias, los modos críticos sobre los textos y los propios textos y sus artífices (Fariña 10). En el 
contexto chileno, el estudio de estas relaciones cuenta con una trayectoria importante que se consolida a partir de los años ochenta, época en la que Darcie Doll identifica dos focos de interés: “A. La reflexión sobre su objeto de estudio: la diferencia de la escritura femenina y/o discursos producidos por mujeres, y, B. La reflexión crítica acerca del canon literario y discursivo y las instituciones hegemónicas tradicionales que lo sostienen" ("Escritura/ literatura" 84). Estos focos, según sus apreciaciones, se interrelacionan con las categorías de género sexual o identidades genérico-sexuales, los métodos de recepción, la relación entre lo político y lo estético y la especificidad de la escritura en el contexto local.

En este escenario, la crítica literaria feminista ha trazado rutas conceptuales y figuraciones teóricas para sistematizar el conocimiento acumulado en torno a la literatura escrita por mujeres. Aquí el concepto de "genealogía" constituye un campo de acción relevante en diálogo con la política feminista contemporánea. Dicho concepto refiere a una apuesta metodológica que posibilita la recuperación de los saberes de las mujeres y la producción de discursos y prácticas de la vida social, poniendo en el centro sus experiencias, así como también el desvelamiento de los artilugios del poder patriarcal según determinados contextos (Restrepo 35-37). Del mismo modo, asume un carácter no lineal para tomar distancia de las genealogías patriarcales, pues "la genealogía feminista no es un recuento de hechos del pasado. Es una revisión crítica del presente mediante la lectura contextual de las condiciones de emergencia y devenir de ideas, concepciones, prácticas y experiencias del sujeto mujeres y la praxis feminista" (Restrepo 36).

Desde este posicionamiento metodológico es posible construir archivos sobre las aportaciones de las mujeres al campo intelectual y ponerlos en diálogo mediante "el ejercicio de ubicación espacio-temporal de las otras que no vivieron nuestro presente" (Restrepo 35), pero a quienes urge convocar mediante la activación de contramemorias (Braidotti 212), proceso que Foucault denominó también como la "insurrección de los saberes sometidos" en contra de los efectos del saber centralizador (219). Esta resignificación feminista de la genealogía foucaultiana es, para el campo de los estudios literarios, una estrategia clave para abandonar la idea hegemónica de tradición literaria entendida como un conjunto de obras canonizadas (Doll, "Escritura/ literatura" 87). Hacer genealogía es estudiar críticamente las relaciones entre textos y efectuar el seguimiento de las estructuras de poder, las que, según Rodríguez, determinan quién tiene acceso al saber, quién puede producirlo, qué valoraciones rigen su transmisión, desde qué instituciones se producen 
los discursos o cuál es el criterio de legitimidad (cit. en Doll, "Escritura/ literatura" 88).

Castro-Gómez, por su parte, advierte que, al centrarse en las estrategias de poder, el método genealógico tensiona el presente para pensar no tanto en lo que nos une, sino mostrar los antagonismos, dilemas, juegos de poder, las rupturas, los vacíos, las fisuras y las líneas de fuga (cit. en Espinosa 2013). Es decir, la genealogía como método es clave para identificar los procesos de conformación de determinados marcos interpretativos y las prácticas que les constituyen. En términos feministas, por ejemplo, esto implica repensar sus propósitos y orígenes, pues, como reflexiona Yuderkys Espinosa, la política feminista necesita interrogar constantemente sus marcos epistémicos y hacer "genealogía de la experiencia" (2017). Este ejercicio autorreflexivo tiene especial significado para los feminismos contemporáneos en un escenario marcado por la aparición de las perspectivas latinoamericanas decoloniales, que buscan repensar el sujeto político del feminismo en términos de las intersecciones entre género, clase, raza y sexualidad. En palabras de Espinosa, "[e]1 proyecto genealógico busca indagar los discursos a los que hemos adherido sobre la sexualidad, el género, el sujeto sexo-genérico, y las maneras en que hemos aplicado estos discursos para pensar "lo latinoamericano" (2015). En este sentido, la genealogía, además de funcionar como contramemoria y contribuir a la legitimación de los saberes producidos por los sujetos subalternizados, es un dispositivo crítico para identificar las lógicas de poder que contribuyen a la conformación de ciertos discursos.

A partir de este marco conceptual, se ratifica el potencial de la labor genealógica en el campo de la teoría y crítica literaria feministas: por una parte, posibilita reconocer el valor epistémico de las alianzas entre prácticas literarias y políticas, y, por otra, recoger los acuerdos y contradicciones de los campos de acción de los feminismos y sus procesos de articulación en su devenir histórico ${ }^{2}$. En este quehacer, son numerosos los trabajos que actualmente pueden servir de ejemplo: las investigaciones realizadas por Andrea Kottow han advertido que las primeras feministas formalmente organizadas en el Chile de comienzos del siglo XX fueron escritoras (152).

$2 \quad \mathrm{Al}$ asumir las especificidades de los movimientos feministas en Chile, este trabajo coincide con las reflexiones de Fina y Figueroa (52), quienes prefieren usar los conceptos de proceso y campo de acción por sobre el de "olas". Para las autoras, estos conceptos permiten comprender los movimientos feministas en su constante construcción y transformación, a partir de diversas estrategias y espacios de lucha. 
Antecedente relevante, pues corrobora que las figuras de la escritora y de la pensadora y/o activista feminista se dieron conjuntamente en una serie de figuras de central importancia para estas primeras movilizaciones (Kottow 153): Inés Echeverría (Iris), Delia Rojas (Delie Rouge), Elvira Santa Cruz Ossa (Roxane), Amanda Labarca y Marta Vergara, entre otras. Si bien Kottow no plantea su trabajo desde lo genealógico, la revisión de estas autoras en función de tres ejes de análisis -1) la comprensión de la escritura que estas mujeres despliegan en sus discursos, 2) la visión de la mujer que los escritos de las autoras constituyen y 3) la visión del concepto de feminismo que puede desprenderse de los escritos (157)-, posibilita "configurar un mapa de los primeros discursos feministas en Chile" (ibid.). Así, este gesto cartográfico dialoga con lo genealógico para trazar posiciones y sentidos de la política: mientras unas autoras fueron cercanas a un feminismo de élite $\mathrm{y}$ aristocrático-cristiano, otras practicaron un feminismo mesocrático-laico enfocado en el sufragismo. Estas fricciones y contradicciones evidencian, asimismo, un proyecto emancipatorio con sus propios derroteros. Doll llama a estas escritoras "precursoras" y sintetiza sus tensiones de la siguiente forma:

La mayoría de estas escritoras asumen ideas feministas o proyectan una perspectiva especialmente enfocada en las problemáticas de las mujeres, y aun cuando algunas se acerquen a ideas más conservadoras, marcan un importante hito en la trayectoria de las mujeres por obtener legitimidad en el espacio social. A través del feminismo realizan una crítica de la cultura que abarca el espacio privado y el público, y es, probablemente, el núcleo más importante del avance en su inserción como intelectuales ("Escritoras chilenas" 28).

Para continuar con esta línea de periodización, Doll denomina modernas a autoras como Marta Brunet, Olga Acevedo (Beatriz Acuña), Mary Yan (María Flora Yáñez), Winétt de Rokha (Luisa Anabalón Sanderson), María Monvel (Tilda Brito Letelier), Gloria Moreno (Esther Irarrázabal de Larraín) y Gabriela Mistral. Estas escritoras mantuvieron alianzas intelectuales con las precursoras, pero enfocaron sus intereses en la demanda por la igualdad en los derechos políticos. En este escenario, Amanda Labarca, ubicada en medio de estos dos grupos, tuvo un rol importante en la consolidación de organizaciones culturales, cuyo proyecto político se sustentó en la necesidad de una formación/educación feminista para las mujeres (Doll, "Escritoras chilenas" 29-35). Finalmente, Doll cierra estas trayectorias destacando a las 
escritoras profesionales, entre estas Magdalena Petit, Magda Arce, Georgina Durand, Lenka Franulic, María Carolina Geel y Pepita Turina, quienes representaron la inscripción definitiva de las mujeres en la escena literaria nacional ("Escritoras chilenas" 26).

Sobre las escritoras profesionales, mencionadas por Doll, Kottow y Traverso publicaron recientemente Escribir y tachar. Narrativas escritas por mujeres en Chile (1920-1970) (2020). En este ensayo las investigadoras realizan una relectura de obras escritas en este periodo con el fin de crear nuevas constelaciones en función de temas, tópicos y problemas (Kottow y Traverso 13); aspectos fundamentales para comprender las aportaciones de las autoras del conocimiento literario. Para alcanzar dichos propósitos, el texto ofrece un análisis de las condiciones de ingreso de las escritoras al mundo profesional de la literatura, concentrándose en los dispositivos de poder/saber del apartado crítico profesionalizado y las estrategias de legitimación del canon.

Debido a que, en el ámbito letrado, la categoría "mujer" designaba no solo a una intrusa, sino a una impostora que fingía saber (Kottow y Traverso 20), las autoras que escribieron desde la reafirmación de su género eran tildadas de "marisabillas" y "sabelotodo", epítetos con los que identificaban a aquellas que tenían el descaro de feminizar la escritura. Como respuesta a estas lógicas de modelamiento patriarcal, la escritura se concibió entre la ambivalencia de ser autorizada por los críticos y la decisión de caer en el "silencio feminista", como lo planteara Julieta Kirkwood en relación con, por ejemplo, las escritoras del cincuenta. Estas escritoras, señalan Kottow y Traverso, "no asumen el activismo feminista como forma de lucha contra el sistema patriarcal que consideran opresor; por el contrario, intentarán excusarse y de alejarse de cualquier adscripción "feminista" (24). Esto, explican más adelante, "obedece a la necesidad y al deseo de las autoras por verse incluidas en el canon literario nacional" (ibid.), pues las experiencias de las feministas de comienzos de siglo habían demostrado que, pese a sus acciones conducentes a ampliar la participación de las mujeres en todos los ámbitos, no habían logrado el respeto de los críticos. En este contexto, en consecuencia, las prácticas literarias y feministas entrarán en tensión, produciéndose giros sobre sus demandas sociales y, con el tiempo, una reconfiguración de su sujeto político ${ }^{3}$.

3 Como nota al pie y para cerrar este apartado, vale la pena y la justicia epistémica nombrar otros importantes trabajos que, ya sean de corte genealógico explícito o a través 


\section{SENTIDOS POSIBLES: EL PENSAMIENTO FEMINISTA DE MERCEDES VALDIVIESO}

El abordaje genealógico de la conjunción literatura/feminismo ha posibilitado, hasta el momento, instalar algunas preguntas: ¿qué política feminista encarna La brecha? ¿Cómo representa ese feminismo en el relato y cuál es su condición de posibilidad? A partir de estas interrogantes, los siguientes párrafos ofrecen un análisis considerando dos conceptos articuladores de las ideas de Valdivieso: el cuerpo y la maternidad. Esta lectura se realiza a partir de una puesta en diálogo con obras de Diamela Eltit y Lina Meruane, autoras ubicadas en la narrativa reciente o de la postdictadura, periodo que, según Areco (288), abarca desde 1990 hasta la actualidad.

Para dar sustento a dicha puesta en diálogo, se recogen las reflexiones de Eliana Ortega: "la importancia de escribir crítica literaria es la de poner las obras en relación; es decir, leerlas en su compleja relación textual, social, histórica, filosófica, estética, con textos anteriores y contemporáneos, y con otros discursos culturales; pero por, sobre todo, me interesa que estos textos y el mío se relacionen con sus lectores" (29). Es decir, se concibe el trabajo crítico-interpretativo feminista como un espacio de encuentros intertextuales, tensiones y convergencias en perspectiva histórica no lineal, con el fin de rastrear los aportes y reflexiones de las mujeres al campo de la política feminista y las prácticas literarias.

de otras estrategias metodológicas, han rastreado y ubicado a las escritoras en los mapas y memorias del feminismo chileno y latinoamericano. Entre estos destacan: Siete escritoras chilenas (1949) de María Carolina Geel, Escribir en los bordes. Congreso Internacional de Literatura Femenina Latinoamericana 1987 (1990) compilado por Carmen Berenguer et.al., Lo que se hereda no se hurta. Ensayos de crítica literaria feminista (1996) de Eliana Ortega, Lengua vibora: producciones de lo femenino en la escritura de mujeres chilenas (1998) de Raquel Olea, Memorias y nomadias: géneros y cuerpos en los márgenes del Posfeminismo (2004) de Ana Forcinito, Entre la casa y la ciudad. La representación de los espacios público y privado en novelas de narradoras latinoamericanas de primera mitad del siglo XX(2016) de Natalia Cisterna y Paso de pasajes: crítica feminista (2018) de Gilda Luongo. 


\section{EL CUERPO}

El cuerpo, categoría epistémica central de los feminismos, ha sido entendido como una entidad socializada y codificada culturalmente; "el sitio de intersección de lo biológico, lo social y lo lingüístico, esto es, del lenguaje entendido como el sistema simbólico fundamental de una cultura" (Braidotti 16). Este carácter social, según Torras, implica que ya no puede ser pensado como una materialidad previa e informe, ajena a la cultura y a sus códigos, pues "tiene una existencia performativa dentro de los marcos culturales (con sus códigos) que lo hacen visible" (20). Así, más que tener o ser un cuerpo, nos convertimos en uno mediante una serie de negociaciones y "coordenadas que nos hacen identificables, reconocibles, a la vez que nos sujetan a sus determinaciones de ser, estar, parecer o devenir" (ibid.). En este devenir, advierte Torras, el cuerpo asume su carácter reproductivo marcado por la irrupción inevitable de una diferencia (el sexo-género, la raza, la clase), pero también es productivo en la medida en que siempre existen desajustes a dicho orden, desde donde surge su capacidad de resistencia y transformación.

En La brecha, este cuerpo se inscribe en la cultura burguesa de la segunda mitad del siglo XX. La personaje sin nombre es signada según los códigos en razón de su sexo/género y las determinaciones de su clase: un cuerpo femenino que se educa, contrae matrimonio, materna y ocupa el espacio de la casa. Esta fórmula, sin embargo, se va desarticulando en la medida en que la protagonista/narradora anula su matrimonio, aborta en su segundo embarazo, concierta con amantes, busca trabajo y construye su propio hogar. Estos contrasentidos, como bien señala Coddou, pueden ser interpretados como el "desborde de los límites aparentemente intraspasables entre la esfera pública y la esfera privada, entre el mundo de la cotidianidad habitual y el de la acción en marcos más amplios" (44), motivo que coincide con las reflexiones feministas que, para ese entonces, a nivel global, inician un proceso de replanteamiento después de la efervescencia sufragista que se traducirá, entre varias otras demandas, en el cuestionamiento de los roles de género y la lucha por los derechos sexuales y reproductivos con la aparición de la píldora anticonceptiva.

La brecha recoge estas preocupaciones en un momento de latencia para Chile, pues, como señala Arcos, a partir de Julieta Kirkwood, no es sino hacia los setenta que el movimiento feminista se reactiva en el marco de las luchas en contra de las dictaduras cívico-militares (50). Por lo tanto, la novela constituye un intersticio que, como sugiere Olea, explora otros espacios y 
"busca nuevas identidades emergidas desde el propio deseo" (103); deseo a través del cual desvía la norma de género y construye otras posibilidades de existencia.

En relación con este último aspecto y con el propósito de poner en diálogo este tratamiento de lo corpopolítico, vale la pena mencionar algunas de las reflexiones que, décadas después, ha realizado la escritora Diamela Eltit a través de sus narraciones. En Los vigilantes, por ejemplo, madre e hijo habitan el espacio de la casa asediados por un padre que, aunque ausente, tiene pleno poder sobre ellos. Como sugiere Barrientos, la vigilancia es representada por medio de esta ausencia que solicita explicaciones de la acción de la familia y que obliga a la madre a escribir cartas a diario. A través de esta demanda, "el vigilante obliga a la elaboración de un discurso logocéntrico, masculino y lineal, ya que la madre debe informarle a través de las cartas sobre su rutina diaria" ("Cartografías quebradas" 27). Por el contrario, en La brecha, la protagonista sale de casa una vez que es consciente de la imposibilidad de su relación con Gastón y la escritura adquiere sentidos liberadores. Así lo reflexiona al plantear su deseo de escribir un libro, mientras aborda un avión que la llevará a Nueva York en busca de mercadería para dar marcha a un negocio de venta de ropa.

Desde estos entramados ficcionales emergen posicionamientos feministas que, aunque distanciados por sus contextos de producción, confluyen en una idea fundamental: el cuerpo es el espacio de resistencia y agencia para las mujeres. En Los vigilantes, la madre, si bien escribe según el mandato patriarcal, constituye una figura de oposición, lo que se evidencia en las desviaciones que van sufriendo sus cartas hasta culminar en un discurso sin sentido y balbuceante, fuera del logos masculino y convertida en "un cuerpo abyecto que provoca la ruptura y la subversión" (Barrientos, "La resistencia orgánica" 153). Asimismo, en Valdivieso, la protagonista, también madre, toma distancia de los mandatos y prescripciones sociales para invertir el metarrelato de la femineidad, asumiendo que lo que le constituye como mujer no es el cumplimiento y asimilación de la norma de género, sino las múltiples posibilidades de encarnar el ser mujer.

\section{LA MATERNIDAD}

La maternidad y sus diversas estrategias de representación han sido una constante en las prácticas literarias de las mujeres en Chile. Así lo ha evidenciado 
Lorena Amaro, quien analizó la producción narrativa de los últimos treinta años concluyendo que en las escrituras de dictadura y postdictadura existe una serie de ficciones sobre lo materno que difieren en sus retóricas y sus efectos políticos (35). Autoras como Diamela Elitit y Pía Barros, por ejemplo, han pensado lo matero desde la estética abyecta y grotesca para reflexionar sobre los efectos del poder de las leyes patriarcales y la represión dictatorial.

En contraste, las escritoras Claudia Apablaza y Lina Meruane, ubicadas en el periodo de 2000 a 2018, "buscan definir o redefinir la experiencia maternal desde perspectivas porosas, fluidas, en que ya no tiene cabida el 'grotesco anatómico' como crítica fuerte de la institución maternal" (Amaro 25), sino las múltiples formas de experimentar la maternidad en un contexto caracterizado por la liquidez, la transterritorialidad y el avance de la tecnología; momento en que la maternidad forma parte del juego de consumo y las madres se ven enfrentadas a las exigencias de estas nuevas condiciones de vida (ibid.). Estos antecedentes son relevantes para situar las diferencias y convergencias entre las escritoras recientes y las ideas sobre la maternidad planteadas por Valdivieso.

En La brecha lo materno tiene dos momentos claves: el primero refiere a la experiencia de ser madre en el marco de su relación matrimonial, y el segundo a cuando decide abortar en medio del fracaso de esta unión. En ambos, la narradora establece una serie de reflexiones que deconstruyen la institucionalidad materna; proceso de racionalización que inicia cuando nace su hijo Sebastián: "Me dolió, me desgarró, me aplicaron calmantes. Nació sano, hermoso. Lo vi al volver de la anestesia un par de horas después. El cansancio era muy grande para tener manifestaciones de alegría. Y estaba contenta. Libre otra vez; al menos, sola con mi propio cuerpo" (Valdivieso 33). El nacimiento, como reflexiona Guerra, simboliza el "proceso de liberación que implica ir contra las normas de la sociedad para buscar su propia identidad" ("Feminismo y subversión" 8). Este inicio de la trayectoria individual marcará, además, el camino para abortar en su segundo embarazo; acto radical y paradigmático no solo a nivel temático-literario, sino también social, pues converge con una de las demandas fundamentales del feminismo en Chile, hasta hoy sin posibilidad de legislarse.

Al mismo tiempo, estas ideas se relacionan con dos categorías clave para los feminismos contemporáneos: identidad y subjetividad. Como reflexiona Lagos, a partir de Butler, estos procesos de subjetivación y construcción de identidades individuales reafirman la imposibilidad de pensar al sujeto como algo universal, original y estable (283). Por el contrario, tal como lo 
demuestra la novela, la protagonista asume en apariencia los mandatos, pero los transgrede sin buscar la redención, reafirmando su capacidad de agencia.

Esta búsqueda de identidad y la posibilidad de decidir sobre la experiencia materna se construyen a partir de lo que Nora Domínguez entiende como "salirse de madre", vale decir, un proceso de renovación simbólica que busca alterar e interrogar los sitios naturalizados de la maternidad, cuyo principio es la ausencia de la voz de la madre ("Salidas de madre" 165). En este proceso de reinvención, la novela transgrede los imaginarios en torno al género femenino mediante el uso de la primera persona. Domínguez comprende la potencia de esta estrategia discursiva del siguiente modo: "Cuando las madres enuncian su propio relato aparecen las versiones fluctuantes, proliferantes y desestabilizadoras del género. Construcciones que trabajan al género en su versión madre y no a la madre como única versión del género" ("El relato de la madre" 178). Si la maternidad es una institución y narrativa hegemónica, la incorporación de la voz propia y el mundo interior de la protagonista son estrategias para desmontar esos discursos, revelando el carácter construido e inestable de los atributos y roles de género.

En relación con esto último, Guerra advierte que en La brecha existe una conciencia declarada sobre la injusta desigualdad de género que privaba a la mujer de la participación activa en la sociedad ("Prólogo" 2). Por esta razón, las acciones disruptivas de la protagonista contribuirán no solo a salirse de los mandatos (salirse de madre), sino a instalar un discurso y evidenciar los procesos de subalternización de las mujeres y sus efectos materiales. Tal como reflexiona Guerra, Valdivieso transforma a la protagonista en una mujer capaz de identificar 'los prejuicios y mitologías de 'lo femenino' como un dispositivo del poder patriarcal que, a través de una normativa plagada de prohibiciones, oculta con su uso de eufemismos que elogian la pureza de la virginidad o la maternidad sublime, su objetivo de mantener a la mujer en su lugar subalterno" (ibid.). En este sentido, construye un discurso feminista que, en sus fundamentos, coincide con las reflexiones de autoras como Gayatri Spivak y su teoría de la subalternidad, la que no solo ha contribuido a comprender las asimetrías de poder en el marco del colonialismo, sino también los efectos de esas prácticas de dominación en los sujetos feminizados.

Meruane, por su parte, interroga la maternidad desde su posición como escritora/creadora que ha decidido no tener hijos. A través de la ironía, en Contra los hijos plantea una resistencia al "entronizamiento del mercado en la conformación de estas figuras adversas a la liberación femenina" (Amaro 20) y a las nuevas exigencias productivas del mercado, mediante las cuales se 
les exige a las mujeres ser "súper madres", "madres-superior-en-todo". Como lo explica Amaro, no se trata literalmente de estar "contra los hijos", sino de cuestionar la máquina productiva que, en seno de la sociedad neoliberal, reafirma el mandato maternal.

De este modo, ambas autoras, mediante estrategias y géneros diversos, destejen los códigos maternizantes para privilegiar la libertad de decisión. Mientras que Meruane recurre al ensayo y a la documentación con el fin de interrogar las fricciones entre el ejercicio materno y la posibilidad de desarrollarse profesionalmente como escritora, Valdivieso construye una trama novelesca a partir de una sujeto que busca liberarse y colectivizar su experiencia. En relación con esto último, son relevantes las reflexiones que Pélage realiza en torno a estas estrategias enunciativas: si bien parece existir coincidencia entre las experiencias de la autora y la narradora (18), se recrea una distancia en la que la narradora dice representar a "cualquier mujer de nuestra generación" (Valdivieso 8). Este ejercicio de colectivización de la voz posibilita entablar un diálogo con las lectoras potenciales y generar procesos de identificación y concientización sobre los efectos del deber ser materno en la vida de las mujeres.

\section{A MODO DE CONCLUSIÓN: APERTURAS Y TENSIONES DE LA BRECHA}

A través de la revisión genealógica de algunos hitos en torno a escritoras del siglo XX se reafirmó el valor de sus aportes a la producción de saberes y memorias feministas. De igual forma, este método posibilitó iluminar algunas de las investigaciones que han rastreado dichos conocimientos; gesto que constituye el actual ethos de la política y la labor crítica: nombrar y articular cultura feminista.

El segundo apartado, enfocado en las representaciones feministas que encarna La brecha, posibilitó identificar algunos puntos de encuentro entre el pensamiento de Valdivieso y las prácticas literarias de autoras recientes. Mediante este diálogo, se concluye que, si bien existen zonas de convergencias en relación con el tratamiento del cuerpo y lo materno, las miradas que conducen a estas reflexiones han sido amplificadas debido a las reconfiguraciones del sujeto político y las diversas estrategias de actuación feminista. Asimismo, fuera de lo obvio - es decir, que estas ampliaciones se deben a las emergencias y especificidades de sus contextos históricos-, interesa mostrar la potencia 
de la activación de contramemorias, pues, cuando muchas de las discusiones en torno a la condición social de las mujeres parecían haberse zanjado, los diálogos entre el pasado y el presente constituyen hoy una de las tácticas para evidenciar la continuidad de "las brechas", pero también para buscar alternativas de desestabilización de las estructuras de poder.

Finalmente, la lectura sobre la novela de Valdivieso deja aperturas y tensiones. De las primeras se han adelantado algunas, especialmente vinculadas al giro paradigmático que significó y que marcó la salida del silencio feminista y el inicio de lo que Nelly Richard hoy llama "revolución cultural del feminismo: un deseo que cuestiona tanto las arquitecturas de poder institucionales como los contratos de lenguaje y representación con los que lo masculino-dominante reparte desigualmente valor y sentido en la sociedad" (315). Este es un giro irreversible, cuya máxima expresión es el mayo feminista de 2018 y su correlato en el estallido social de octubre de 2019. En relación con las tensiones, vale la pena preguntarse por las cuestiones de clase, raza y sexualidad, las que, si bien se tocan tangencialmente en la novela, no logran constituirse en ejes articuladores de una política más compleja, tal como lo plantea actualmente el feminismo decolonial.

\section{BIBLIOGRAFÍA}

Amaro Castro, Lorena. "Maternidades 'líquidas': feminismos y narrativas recientes en Chile". Revista Chilena de Literatura 101, 2020, pp. 13-39.

Arcos Herrera, Carol. "Feminismos latinoamericanos: deseo, cuerpo y biopolítica de lo materno". Debate feminista 55, 2018, pp. 27-58.

Areco Morales, Macarena. "Cartografía de la novela chilena reciente: realismos, experimentalismos, hibridaciones y subgéneros”. Chile en el siglo XXI: ¿nuevos recorridos artísticos, nuevos caminos históricos? Laetitia Boussard y Benoît Santini (eds.), Santiago, Piso Diez Ediciones/MAGO Ediciones, 2013, pp. 288-328.

BARrientos, Mónica. "La resistencia orgánica y la figura del cyborg”. La pulsión comunitaria en la obra de Diamela Eltit. Mónica Barrientos, Pittsburgh, Estados Unidos, Latin American Research Commons, 2019, pp. 153-164.

“Cartografías quebradas y cuerpos marginales en la narrativa de Diamela Eltit".

Debate Feminista 53, 2017, pp. 18-32.

Braidott, Rosi. Feminismo, diferencia sexual y subjetividad nómade. Barcelona, Gedisa S.A, 2004.

Coddou, Marcelo. "Una relectura de La Brecha de Mercedes Valdivieso". Inti: Revista de 
Literatura Hispánica 29, 1989, pp. 39-48.

De Fina González, Débora y Francisca Figueroa Vidal. "Nuevos 'campos de acción política' feminista: Una mirada a las recientes movilizaciones en Chile". Revista Punto Género 11, 2019, pp. 51-72.

Doll, DARCIE. "Escritoras chilenas de la primera mitad del siglo XX: trayectoria en el campo literario y cultural como criterios para una periodización de su producción". Taller de Letras 54, 2014, pp. 23-38.

"Escritura/Literatura de mujeres: crítica feminista, canon y genealogías". Universum 17, 2002, pp. 83-90.

Domínguez, Nora. "Salidas de madre para salirse de madre". Revista Iberoamericana 69, 2003, pp. 165-181.

“El Relato de la Madre”. Travessia Revista de Literatura 29, N³0, 1994/1995, pp. 163-179.

Eltit, Diamela. Los vigilantes. Santiago, Editorial Sudamericana, 1994.

EsPinosa MiÑoso, Yuderkys. "Hacer genealogía de la experiencia: el método hacia una crítica a la colonialidad de la Razón feminista desde la experiencia histórica en América Latina”. Revista Direito Práx 3, N³, 2019, pp. 2007-2032.

FariÑa Busto, M. J. "Feminismo y Literatura. Acerca del canon y otras reflexiones". Revista de Escritoras Ibéricas 16, 2017, pp. 9-41.

Foucault, Michel. Microfisica del poder. Buenos Aires, Siglo XXI Editores, 2019.

Guerra, Lucía. "Prólogo. Independencia política y artística de la mujer en Los ojos de

Bambú de Mercedes Valdivieso". Los ojos de Bambú, Mercedes Valdivieso, Santiago, Ediciones Universidad Alberto Hurtado, 2021. pp. 7-17.

"Feminismo y subversión en La brecha de Mercedes Valdivieso". Literatura

Chilena, Creación y Crítica 3, 1982, pp. 5-9.

Kottow, ANDrea y Ana Traverso. Escribir y tachar. Narrativas escritas por mujeres en Chile (1920-1970). Santiago, Overol, 2020.

KotTow, ANDREa. "Feminismo y femineidad: escritura y género en las primeras escritoras feministas en Chile". Atenea 508, 2013, pp. 151-169.

LAgos, María InÉs. Hechura y confección. Escritura y subjetividad en las narraciones de mujeres latinoamericanas. Santiago, Editorial Cuarto Propio, 2009.

Leunda, Ana InÉs. "Heroínas en la novelística de M. Valdivieso: entre cuerpos, metáforas y culturas". Rétor 1, 2015, pp. 65-87.

Meruane, Lina. Contra los hijos. Santiago, Penguin Random House, 2018.

OlEA, RAQUel. "Escritoras de la generación del cincuenta: claves para una lectura política". Universum 2, 2010, pp. 101-116.

"La palabra maldita". Lengua víbora. Producciones de lo femenino en la escritura 
de mujeres chilenas. Raquel Olea, Santiago, Editorial Cuarto Propio, 1998, pp. 101-113. Ortega, Eliana. Lo que se hereda no se hurta. Ensayos de crítica literaria feminista. Santiago, Editorial Cuarto Propio, 1996.

Pélage, Catherine. "Una confidencia desafiante. La brecha de Mercedes Valdivieso". La palabra y el hombre 18, 2011, pp. 17-21.

Restrepo, Alejandra. "La genealogía como método de investigación feminista". Lecturas críticas en investigación feminista. Norma Blazquez Graf y Martha Patricia Castañeda Salgado (coord)., Ciudad de México, Centro de Investigaciones Interdisciplinarias en Ciencias y Humanidades, Programa de Posgrado en Estudios Latinoamericanos, 2016, pp. 23-41.

Richard, Nelly. "La insurgencia feminista de mayo 2018 en Chile". Zona de tumultos:

Memoria, arte y feminismo. Textos reunidos de Nelly Richard: 1986-2020. Buenos Aires, CLACSO, 2021, pp. 311-327.

Torras, Meri. "El delito del cuerpo. De la evidencia del cuerpo al cuerpo en evidencia". Cuerpo e identidad. estudios de género y sexualidad 1. Meri Torras (ed.), Barcelona, Ediciones UAB, 2007, pp. 11-36.

Valdivieso, Mercedes. La brecha. Santiago de Chile, Zig-Zag, 1961. 